\title{
Hypoxia Induced by Vascular Damage at High Doses Could Compromise the Outcome of Radiotherapy
}

\author{
EMELY KJELLSSON LINDBLOM ${ }^{1}$, ALEXANDRU DASU ${ }^{2,3}$ and IULIANA TOMA-DASU ${ }^{1,4}$ \\ ${ }^{1}$ Medical Radiation Physics, Department of Physics, Stockholm University, Stockholm, Sweden; \\ ${ }^{2}$ Department of Medical and Health Sciences, Linköping University, Linköping, Sweden; \\ ${ }^{3}$ The Skandion Clinic, Uppsala, Sweden; \\ ${ }^{4}$ Department of Oncology and Pathology, Karolinska Institute, Stockholm, Sweden
}

\begin{abstract}
Background/Aim: This study investigated the impact of temporary vascular collapse on tumour control probability $(T C P)$ in stereotactic body radiotherapy (SBRT), taking into account different radiosensitivities of chronically and acutely hypoxic cells. Materials and Methods: Three-dimensional tumours with heterogeneous oxygenation were simulated assuming different fractions of collapsed vessels at every treatment fraction. The modelled tumours contained a chronically hypoxic subvolume of $30-60 \%$ of the tumour diameter, and a hypoxic fraction $\leq 5 \mathrm{~mm} \mathrm{Hg}$ of $30-50 \%$. The rest of the tumours were well-oxygenated at the start of the simulated treatment. Results: For all simulated cases, the largest reduction in TCP from $97 \%$ to $2 \%$ was found in a tumour with a small chronically hypoxic core treated with 60 Gy in eight fractions and assuming a treatment-induced vascular collapse of $35 \%$ in the well-oxygenated region. Conclusion: The timing of SBRT fractions should be considered together with the tumour oxygenation to avoid loss of TCP in SBRT.
\end{abstract}

For decades, stereotactic body radiotherapy (SBRT) has achieved high levels of local control in many tumour sites. Particularly, SBRT has proven successful in treating tumours where conventional methods have failed, such as non-small cell lung cancer (NSCLC) (1). The success of SBRT has been explained by the higher biological effective dose (BED) that can be achieved with a small number of high-dose fractions (2). However, as is commonly summarised by the so-called 5 Rs of fractionated radiotherapy (repair, repopulation,

This article is freely accessible online.

Correspondence to: Emely Kjellsson Lindblom, Medical Radiation Physics, Karolinska University Hospital Solna, S-171 76 Stockholm, Sweden. Tel: +46 764951628, e-mail: emely.lindblom@fysik.su.se

Key Words: Hypoxia, vascular damage, stereotactic body radiotherapy, SBRT, tumour control probability, TCP, tumour oxygenation. reoxygenation, redistribution, and radiosensitivity) (3), the outcome of a certain combination of the treatment parameters time, dose and number of fractions is, in general, not straightforward to predict. Furthermore, in the context of extreme hypofractionation the classical interpretation of the 5 Rs has been challenged. For example, accelerated repopulation of tumour cells is not expected to occur during the short treatment course in SBRT, and redistribution within the cell cycle is typically also neglected as the high fractional doses are expected to cause permanent cell cycle arrest (4). On the other hand, intra-fraction repair could decrease the biological effectiveness if the delivery time of one large fraction significantly exceeds the repair half-life of tumour cells. Similarly, the limited number of fractions implies a reduced potential for reoxygenation of radioresistant hypoxic tumour cells, which could compromise the outcome of an SBRT treatment (5).

While the intrinsic radiosensitivity is exclusively determined by the biological properties of the tissue and thus invariant to the fractionation schedule, 'new biology' has been suggested to describe the tumour response from high fractional doses in particular (6). In addition to a potential systemic immune response contributing to tumour cell kill at high doses, a secondary effect from vascular damage has also been proposed. This has mostly been discussed as contributing to tumour cell kill in the sense that cells not directly killed by the radiation die as a result of their vascular supply disintegrating. However, the tumour vasculature has also been observed to restore itself following high doses (7), which could make the outcome of a potential vascular effect highly dependent on when subsequent fractions are delivered during an SBRT treatment course. For example, if sufficient time for the vessels to regenerate has elapsed between fractions, the contribution to a secondary form of cell kill would reasonably be much smaller than if the vessels have become permanently degenerated and tumour cells have starved to death. In this context it must be noted that sudden collapses of vessels also occur spontaneously in tumours as 
a result of $e . g$. fluctuations in the interstitial fluid pressure (8). This leads to cells becoming acutely deprived of oxygen (acutely hypoxic) and hence resistant to radiation, which could equally be expected to result from a radiation-induced vascular collapse. In contrast to chronically hypoxic cells, these cells are metabolically active and likely represent the most resistant form of hypoxia, posing a significant threat to the success of radiotherapy (9-11). Thus, if the time between two SBRT fractions is neither enough for damaged vessels to regenerate, nor for the acutely hypoxic tumour cells to become chronically hypoxic and starved, a decrease in the biological effectiveness of the treatment could be implied. It was, therefore, the purpose of this study to investigate the impact from inter-fraction vascular collapse induced by high doses per fraction on the outcome of SBRT treatments.

\section{Materials and Methods}

A three-dimensional in silico tumour model with heterogeneous oxygenation was further developed to simulate radiation-induced vessel collapse between SBRT fractions. Thus, for the purpose of calculating the distribution of oxygen partial pressure $\left(\mathrm{pO}_{2}\right)$ in the tumour, inter-vessel distance distributions with various fractions of collapsed vessels were generated. For all simulations, the position of the vessels in the tumour was random, allowing for the simulation of fast reoxygenation on the microscale between fractions (12). The simulated tumours had a diameter of $20 \mathrm{~mm}$ and were assumed to have chronically hypoxic subvolume with a large mean intervessel distance of $150 \mu \mathrm{m}$. In the remaining part of the tumour, the mean intervessel distance at the start of the treatment was $100 \mu \mathrm{m}$ (Figure 1A). Two sizes of the chronically hypoxic subvolume were considered: $6 \mathrm{~mm}$ and $12 \mathrm{~mm}$ in diameter.

SBRT treatments with a homogeneous dose of 60 Gy delivered in 3,5 or 8 fractions were simulated. For the treatment fractions following the first one, different scenarios with regards to the fraction of collapsed vessels were assumed. On the one hand, it was assumed that any vasculature that had been depleted by the previous fraction had been successfully restored by the time of the subsequent fraction. On the other hand, it was assumed that $35 \%$ of the vessels were collapsed at every fraction following the first (Figure 1B). For the latter case, a homogeneous boost dose of $20 \%$ higher dose ( $72 \mathrm{~Gy}$ ) corresponding to the maximum dose in a typical SBRT treatment was also considered to the chronically hypoxic subvolume (Figure 1C).

The simulated treatments were evaluated by calculating the tumour control probability (TCP) as the product of the individual TCPs in each voxel. The TCP in each voxel was in turn calculated based on an assumed homogeneous density of clonogenic cells at the start of the treatment, and the surviving fraction in each voxel at the end of the treatment taking into account the $\mathrm{pO}_{2}$ at each treatment fraction (12). Based on the hypothesis that chronically hypoxic cells are also energy-depleted and more sensitive than acutely hypoxic cells, different expressions for calculating the surviving fraction were used depending on the simulated $p \mathrm{O}_{2}(10,13)$. Thus, in voxels belonging to the chronically hypoxic subvolume the surviving fraction $S F_{\text {core }}$ after dose $d$ was calculated as:

$$
S F_{\text {core }}=\exp \left(-\frac{\alpha_{S}}{O M F} \cdot d-\frac{\beta}{O M F} \cdot d^{2}\right)
$$

where OMF is the oxygen modifying factor calculated as in previous publications (12), and $\alpha_{S}$ and $\beta$ are the radiosensitivity parameters. In voxels belonging to the acutely hypoxic rim, the following expressions were used:

$$
\begin{aligned}
& S F_{\text {rim }} \\
& = \begin{cases}\exp \left\{-\alpha_{R} \cdot\left[1+\left(\frac{\alpha_{S}}{\alpha_{R}}-1\right) \cdot e^{-\frac{d}{D_{C}}}\right] \cdot d-\beta \cdot d^{2}\right\} & \text { for } \mathrm{O}_{2} \geq 30 \mathrm{mmHg} \\
\exp \left\{-\frac{\alpha_{R}}{O M F} \cdot\left[1+\left(\frac{\alpha_{S}}{\alpha_{R}}-1\right) \cdot e^{-\frac{d}{D_{C} \cdot O M F}}\right] \cdot d-\frac{\beta}{O M F} \cdot d^{2}\right\} & \text { for } p \mathrm{O}_{2}<30 \mathrm{mmHg}\end{cases}
\end{aligned}
$$

The parameters used for the simulations were $\alpha_{S}=0.66 \mathrm{~Gy}^{-1}$, $\alpha_{R}=0.33 \mathrm{~Gy}^{-1}, \alpha_{R} / \beta=10 \mathrm{~Gy}, \quad \beta=0.033 \mathrm{~Gy}^{-2}, D_{C}=0.27 \mathrm{~Gy}$, $O M F_{\text {max }}=3$ and $N=10^{6}$ cells, where $N$ corresponds to the number of clonogenic cells at the beginning of the treatment.

\section{Results and Discussion}

Examples of cross-sections through the simulated tumours can be seen in Figure 1. For both sizes of the chronically hypoxic subvolume, the hypoxic fraction $\leq 5 \mathrm{~mm} \mathrm{Hg}$ was $49 \%$ in the core, and $0 \%$ in the rim assuming no vessel collapse. With $35 \%$ collapsed vessels, the hypoxic fraction in the rim increased to $15 \%$. At the beginning of the treatment, the total hypoxic fraction for a tumour with a small and large chronically hypoxic subvolume respectively was 3\% and $15 \%$. For a $35 \%$ vessel collapse, the corresponding numbers increased to $36 \%$ and $41 \%$ respectively.

For all fractionation schedules considered and for both sizes of the chronically hypoxic subvolume, the TCP was reduced when $35 \%$ of the vessels were collapsed after the first fraction with the largest reduction observed for the eight-fractions schedule (Table I). A boost dose of $20 \%$ could only completely counteract the decrease in TCP for the three-fractions schedule, while for the eight-fractions schedule there was no difference in TCP with or without the boost dose. For the five-fractions schedule, a similar reduction in TCP was observed when comparing the case of no vessel collapse with a vessel collapse of $35 \%$, as when the size of the chronically hypoxic subvolume was doubled. For the eight-fractions schedule on the other hand, the TCP for the smaller chronically hypoxic subvolume dropped from $96.8 \%$ to $1.7 \%$ for a $35 \%$ vessel collapse between fractions, while the TCP for the larger subvolume without increased vessel collapse was $83.2 \%$. Furthermore, it is interesting to note that for a homogeneous dose, the highest TCP is obtained for a five-fractions schedule, which could indicate the existence of an optimal combination of time, dose and fractionation with respect to a heterogeneous and dynamic tumour oxygenation. It is important to note that the results of this work are not in contradiction with 
(A)

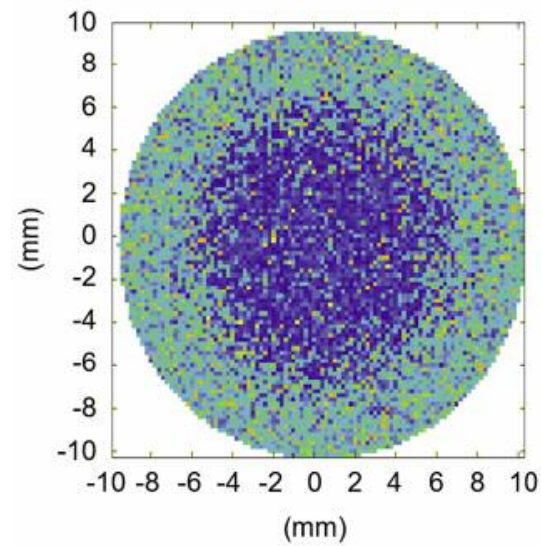

(B)

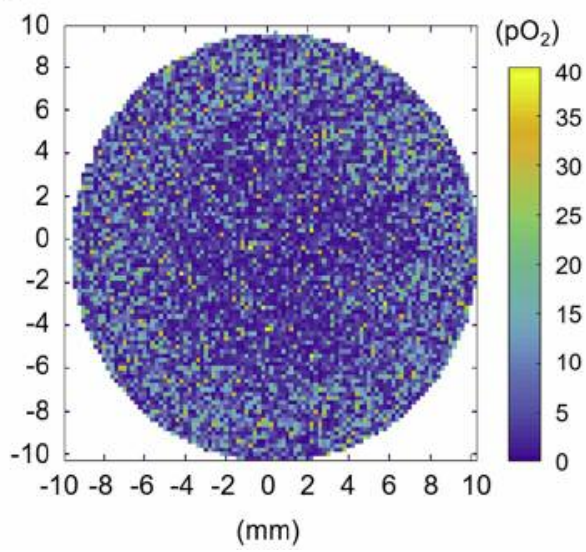

(C)

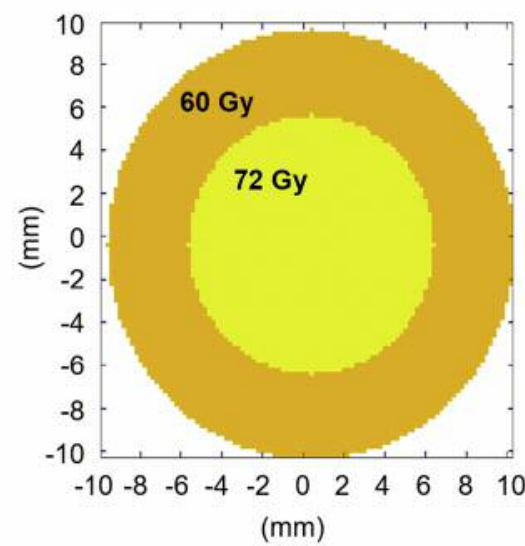

Figure 1. Cross-sections through the simulated tumour with the larger chronically hypoxic subvolume at the beginning of the treatment (A), and with radiation-induced $35 \%$ collapsed vessels (B). The homogeneous dose distribution including a $20 \%$ boost dose is shown in (C).

Table I. Tumour control probability (TCP) for the simulated treatments.

\begin{tabular}{|c|c|c|c|}
\hline \multirow[t]{2}{*}{ Chronically hypoxic subvolume radius $3 \mathrm{~mm}$} & \multicolumn{3}{|c|}{$\mathrm{TCP}(\%)$} \\
\hline & 3 fractions & 5 fractions & 8 fractions \\
\hline No radiation-induced vessel collapse & 83.5 & 99.2 & 96.8 \\
\hline $35 \%$ closed vessels & 79.8 & 93.7 & 1.7 \\
\hline $35 \%$ closed vessels $+20 \%$ boost dose & 99.9 & 94.8 & 1.7 \\
\hline \multirow[t]{2}{*}{ Chronically hypoxic subvolume radius $6 \mathrm{~mm}$} & \multicolumn{3}{|c|}{ TCP $(\%)$} \\
\hline & 3 fractions & 5 fractions & 8 fractions \\
\hline No radiation-induced vessel collapse & 24.0 & 93.3 & 83.2 \\
\hline $35 \%$ closed vessels & 16.5 & 81.0 & 0.6 \\
\hline $35 \%$ closed vessels $+20 \%$ boost dose & 99.4 & 88.5 & 0.6 \\
\hline
\end{tabular}

TCP: Tumour control probability.

the results from Lindblom et al. (13) showing a decrease in isoeffective doses with a larger chronically hypoxic fraction. While in the previous study only a change in the fraction of energy-depleted cells was considered, in the present study the global hypoxic fraction was also increased for a larger chronic subvolume. Furthermore, the pragmatic positioning of the simulated chronic hypoxia in the centre of the tumour does not limit the interpretation of the results to one particular pattern of hypoxia, as the doses applied were homogeneous.

Being a modelling study on relatively unknown phenomena, this work is based on assumptions mainly with regard to the evolution of the tumour oxygenation throughout the treatment. For example, the inter-fraction vessel collapse was assumed to increase only the acutely hypoxic fraction of cells, while an increase of the chronically hypoxic population resulting from cells supported by permanently collapsed vessels moving into the energy-depleted compartment could also occur during the treatment. In addition to considering more complicated dynamics of different hypoxic compartments, an interesting objective for a future study would be to include in the modelling the effect from a systemic immune response, which has also been discussed as highly dependent on the timing of e.g. SBRT fractions (14). Given the limited knowledge so far in this area, the present work contributes to a more nuanced perspective on the clinical impact of tumour hypoxia in stereotactic body radiotherapy. 


\section{Conclusion}

Including both tumour hypoxia and the timing of treatment fractions in stereotactic body radiotherapy treatment planning could increase the probability of cure and help prevent unfavourable fractionation schedules.

\section{Conflicts of Interest}

The Authors have no conflict of interests to declare.

\section{Authors' Contributions}

EKL performed the simulations and wrote the manuscript. AD and ITD produced input data for the simulations and reviewed the manuscript.

\section{Acknowledgements}

Financial support from the Cancer Research Funds of Radiumhemmet (Solna, Sweden) is gratefully acknowledged.

\section{References}

1 Baumann P, Nyman J, Hoyer M, Wennberg B, Gagliardi G, Lax I, Drugge N, Ekberg L, Friesland S, Johansson KA, Lund JA, Morhed E, Nilsson K, Levin N, Paludan M, Sederholm C, Traberg A, Wittgren L and Lewensohn R: Outcome in a prospective phase II trial of medically inoperable stage I nonsmall-cell lung cancer patients treated with stereotactic body radiotherapy. J Clin Oncol 27(20): 3290-3296, 2009. PMID: 19414667. DOI: $10.1200 / J C O .2008 .21 .5681$

2 Onishi H1, Araki T, Shirato H, Nagata Y, Hiraoka M, Gomi K, Yamashita T, Niibe Y, Karasawa K, Hayakawa K, Takai Y, Kimura T, Hirokawa Y, Takeda A, Ouchi A, Hareyama M, Kokubo M, Hara R, Itami J and Yamada K: Stereotactic hypofractionated high-dose irradiation for stage I nonsmall cell lung carcinoma: clinical outcomes in 245 subjects in a Japanese multiinstitutional study. Cancer 101(7): 1623-1631, 2004. PMID: 15378503. DOI: $10.1002 / \mathrm{cncr} .20539$

3 Mayles P, Nahum A and Rosenwald JC (eds): Handbook of Radiotherapy Physics: Theory and Practice. Boca Raton, CRC Press, Taylor \& Francis Group, 2007.
4 Kim MS, Kim W, Park IH, Kim HJ, Lee E, Jung JH, Cho LC and Song CW: Radiobiological mechanisms of stereotactic body radiation therapy and stereotactic radiation surgery. Radiat Oncol J 33(4): 265-275, 2015. PMID: 26756026. DOI: 10.3857/ roj.2015.33.4.265

5 Lindblom E, Dasu A and Toma-Dasu I: Optimal fractionation in radiotherapy for nonsmall cell lung cancer - a modelling approach. Acta Oncol 54(9): 1592-1598, 2015. PMID: 26217986. DOI: 10.3109/0284186X.2015.1061207

6 Brown JM, Carlson DJ and Brenner DJ: The tumor radiobiology of SRS and SBRT: are more than the 5 Rs involved? Int J Radiat Oncol Biol Phys 88(2): 254-262, 2014. PMID: 24411596. DOI: 10.1016/j.ijrobp.2013.07.022

7 Park HJ, Griffin RJ, Hui S, Levitt SH and Song CW: Radiationinduced vascular damage in tumors: implications of vascular damage in ablative hypofractionated radiotherapy (SBRT and SRS). Radiat Res 177(3): 311-327, 2012. PMID: 22229487.

8 Ljungkvist AS, Bussink J, Kaanders JH and van der Kogel AJ: Dynamics of tumor hypoxia measured with bioreductive hypoxic cell markers. Radiat Res 167(2): 127-145, 2007. PMID: 17390721.

9 Vaupel P, Kallinowski F and Okunieff P: Blood flow, oxygen and nutrient supply, and metabolic microenvironment of human tumors: a review. Cancer Res 49(23): 6449-6465, 1989. PMID: 2684393.

10 Denekamp J and Dasu A: Inducible repair and the two forms of tumour hypoxia - time for a paradigm shift. Acta Oncol 38(7): 903-918, 1999. PMID: 10606420.

11 Zolzer F and Streffer C: Increased radiosensitivity with chronic hypoxia in four human tumor cell lines. Int J Radiat Oncol Biol Phys 54(3): 910-920, 2002. PMID: 12377345.

12 Toma-Dasu I and Dasu A: Modelling tumour oxygenation, reoxygenation and implications on treatment outcome. Comput Math Methods Med 2013:141087, 2013. PMID: 23401721. DOI: $10.1155 / 2013 / 141087$

13 Lindblom E, Toma-Dasu I and Dasu A: Accounting for two two forms of hypoxia for predicting tumour control probability in radiotherapy: An in silico study. Adv Exp Biol 1072: 183-187, 2018. PMID: 30178343. DOI: 10.1007/978-3-319-91287-5_29

14 Deloch L, Derer A, Hartmann J, Frey B, Fietkau R and Gaipl US: Modern radiotherapy concepts and the impact of radiation on immune activation. Front Oncol 6: 141, 2016. PMID: 27379203. DOI: $10.3389 /$ fonc 2016.00141

Received April 9, 2019

Revised April 20, 2019

Accepted April 22, 2019 